\title{
Abundance of Plaque Microvessels is Associated With Constrictive Remodeling in Angioplastied Human Coronary Arteries
}

\author{
Abdullah Assiri, MD; John P. Veinot, MD; Kirsten Woodend, PhD; \\ Takeshi Kimura, MD*; Masakiyo Nobuyoshi, MD*; \\ Stephen M. Schwartz, PhD**; Edward R.M. O’Brien, MD
}

\begin{abstract}
This study investigated if specific histological features correlate with remodeling in human coronary arteries treated by balloon angioplasty (PTCA). Segments of perfusion-fixed coronary arteries that had undergone antemortem PTCA were obtained from 15 patients and primary atherosclerotic (CAD) lesions obtained from these hearts were used as control lesions. Arterial segments were serially divided to yield 108 sub-segments for PTCA lesions and 38 sub-segments for CAD lesions. A linear regression analysis was used to determine the relationship between 14 histological parameters and an arbitrary index of compensatory arterial enlargement, the external elastic lamina (EEL) index (EELI), defined as the ratio of the area encompassed by the EEL to the sum of the intimal area (IA) + medial area (MA). In PTCA arteries the abundance of plaque microvessels negatively correlated with the EELI $(\mathrm{p}=0.04)$, but in CAD arteries there was no relationship between histology and the EELI. The abundance of plaque microvessels correlates with the magnitude of constriction in coronary artery lesions subjected to PTCA. This study provides descriptive insights into the biology of remodeling in human coronary arteries after angioplasty, and suggests that the endothelium may play an important role. (Jpn Circ J 2001; 65: 429-433)
\end{abstract}

Key Words: Angioplasty; Atherosclerosis; Humans; Remodeling; Restenosis

$A^{\mathrm{r}}$ rterial remodeling refers to a series of structural changes that occur in arteries responding to various physiologic and pathologic stimuli. Unfortunately, the term arterial remodeling is confusing as it holds different meaning in different contexts. For example, arteries may remodel in response to changes in blood flow (eg, during pregnancy or in the neonatal period) or pressure (eg, hypertension)! The term remodeling also refers to the ability of human coronary arteries to undergo adaptive enlargement in response to progressive accumulation of plaque mass. ${ }^{2-5}$ Remodeling is also a key component of the response to arterial injury; however, with quite a different meaning? In animal models of arterial injury, as well as in studies of human coronary arteries that have undergone angioplasty (or percutaneous transluminal coronary angioplasty (PTCA)), 'shrinkage' or constrictive remodeling of the artery wall is a major determinant of lumenal narrowing, whereas neointimal formation plays a minor role in this process? -14 $^{-14}$

How arterial wall constriction is accomplished or why some but not all arteries undergo compensatory dilatation in order to preserve lumen area (LA) is only now beginning to be studied ${ }^{15-17}$ Blood flow and shear stress are known to

(Received October 6, 2000; revised manuscript received January 19, 2001; accepted February 14, 2001)

University of Ottawa Heart Institute, Ontario, Canada, *Kokura Memorial Hospital, Kitakyushu, Japan and **Department of Pathology, University of Washington School of Medicine, WA, USA Mailing address: Edward R. O'Brien, MD, FRCP(C), FACC, Department of Medicine (Cardiology), Vascular Biology Laboratory, University of Ottawa Heart Institute, 40 Ruskin Street, Ottawa, Ontario, Canada K1Y 4W7. E-mail: eobrien@ottawaheart.ca play a critical role in remodeling, and the response of arteries to chronic alterations in blood flow is endothelium dependent ${ }^{18,19}$ For example, Langille and O'Donnell demonstrated in rabbit carotid arteries that decreased blood flow results in narrowing of the vessel diameter that is unchanged with papaverine and likely caused by structural changes in the artery wall ${ }^{18}$ However, when the endothelium is removed from these vessels the response to reduced blood flow is abolished. In atherosclerotic arteries that contain a rich network of endothelial cell-lined microvessels (MV), or vasa vasorum, the role of the endothelium in regulating remodeling may be very important ${ }^{20-22}$

Inadequate remodeling or overt constriction of the artery wall is also an important component of the pathophysiology of restenosis. Using serial intravascular ultrasound imaging of human coronary arteries immediately after successful PTCA as well as months later during follow-up angiography, Mintz et al defined the relative contribution of changes in plaque and arterial cross-sectional areas to late loss of LA! ${ }^{3}$ These investigators discovered that $73 \%$ of the decrease in LA was due to a decrease in external elastic lamina (EEL) area (EELA), while $27 \%$ was due to an increase in intimal area (IA) and medial area (MA). Similarly, we and others have highlighted the contribution of the tissue immediately peripheral to the EEL (ie, the adventitia) to arterial constriction and lumenal narrowing in porcine models of coronary artery balloon injury $16,23,24$ Thus, the direction and magnitude of the change in EEL primarily determines restenosis after balloon angioplasty.

Given the lack of information concerning the biology of remodeling post angioplasty of human arteries, the goal of the present study was to determine if specific histological 
parameters correlate with arterial remodeling post PTCA. We hypothesized that this histological information might be helpful in providing clues to the mechanisms that govern remodeling.

\section{Methods}

\section{Study Patients}

The coronary arteries of 15 patients who had undergone PTCA of one or more arterial segments, but also had other coronary artery segments with de novo atherosclerotic (or CAD) lesions were studied. (These arteries have been described in a previous manuscript that summarized the histology of post-PTCA restenotic lesions 25 ) Consent to use this tissue for research purposes was obtained from patients' relatives and the institutional committee on human research at the Kokura Memorial Hospital, Kitakyushu, Japan approved the research protocol.

At time of autopsy the heart was isolated and the coronary arteries were perfusion-fixed by infusing $10 \%$ glutaraldehyde from the aortic root at a mean pressure of 90-100 $\mathrm{mmHg}$ for $15-20 \mathrm{~min}$; the heart was then immersion-fixed in $20 \%$ formalin for 3 days. After fixation, the epicardial coronary arteries were dissected from the heart and decalcified. Using an antemortem coronary angiogram as a guide, the coronary artery segments of CAD and PTCA lesions were identified using conventional criteria (eg, proximal, mid and distal coronary artery) as well as proximity to branching segments (eg, diagonal or marginal arteries). A total of $10 \mathrm{CAD}$ arterial segments and 22 PTCA segments were identified. All coronary artery segments, which measured approximately $20-30 \mathrm{~mm}$ in length, were further sub-divided into serial cross-sections at $2-3 \mathrm{~mm}$ intervals. A total of 38 CAD sub-segments and 108 PTCA subsegments were studied.

\section{Morphometric Analysis}

Using a computerized image analysis system (Bioscan Optimus, Edmonds, WA, USA) the extent of arterial narrowing was assessed on Verhoeff van Giesson stained tissue slides. Lumen area and the area encompassed by the internal and external elastic laminae (IELA and EELA, respectively) were manually traced. The IA was calculated by subtracting the LA from the IELA, and the MA was the difference between the EELA and IELA.

\section{Histological Parameters}

Two experienced investigators (JPV and EO'B) graded the histological features of slides stained with hematoxylineosin, as well those stained with Verhoeff Van Giesson, using a semi-quantitative grading system for each arterial sub-segment. All parameters were assigned a score from 0 to 3 , corresponding to an abundance of each feature. Three areas of the vessel wall were graded: adventitia, plaque and artery wall. A score of 0 corresponded to an absence of a specific feature, whereas a score of 1 was used if a specific feature was found in up to one-third of the area of the arterial compartment. Scores of 2 and 3 were used if a particular histological feature was present in one- to two-thirds or more than two-thirds of the area of interest, respectively. To quantify discontinuities in the elastic laminae the same scoring system was used to delineate the extent of the circumferential involvement. As well, for lesion eccentricity, a score of 0 was used if the plaque was concentric, whereas scores of 1,2 or 3 were assigned to lesions with mild, moderate and marked degrees of eccentricity, respectively. The following are the features that were graded.

Adventitia Inflammation: area occupied by mononuclear cells; Microvascularity: area occupied by vasa vasorum.

Plaque Inflammation: area occupied by mononuclear cells (excluding foamy macrophages); microvascularity: area occupied by MV; Lipid: area occupied by cholesterol crystals and foamy macrophages; Necrosis: area occupied by pultaceous debris; Calcification: area occupied by mineralized deposits; Thrombosis: area occupied by fresh or organized thrombus; Intimal hyperplasia: area occupied by stellate myofibroblasts; Fibrotic cap: dense fibrous connective tissue component of lesion.

Artery Wall Medial thinning: area of the media that is attenuated relative to the media underlying adjacent normal segments or a normal area of an eccentric atherosclerotic lesion; Disruption of IEL: extent of IEL circumference with discontinuities; Disruption of EEL: extent of EEL circumference with discontinuities; Eccentricity: refers to the location of the plaque mass, and how far off the central arterial axis this mass resides.

Both examiners were blinded to the nature of each slide, and came to a consensus score for each slide. In order to validate the histological scoring process, each investigator independently graded 10 slides using the histological scoring system described. The examiners' scores were identical for the majority of these specimens with the exception of a few minor differences that were easily resolved by further discussion of the histological scoring criteria. The histological parameters were then tabulated and correlated with an arbitrarily assigned remodeling index (EELI), defined as the ratio of the area encompassed by the EEL to the combined IA+MA.

\section{Statistical Analysis}

For each group of specimens the areas for the various arterial wall compartments were expressed as mean values \pm 1 SD. A t-test was used to look for differences in the areas between the CAD and PTCA groups. The relationship between LA and (IA+MA) for each group was determined using linear regression, and the correlation coefficients were compared between groups. A probability value less than 0.05 was considered significant. The slopes of the curves that summarized the relationship between LA and (IA+MA) for both the CAD and PTCA arteries were compared using a t-test. Multiple linear regression analysis was performed to assess the relationship between the various histological parameters and the index of compensatory enlargement for each artery [EELI =EELA / $(\mathrm{IA}+\mathrm{MA})]$. The EELI was designated as the dependent variable and the 14 histological parameters were independent variables. As multiple slides were obtained from each subject there was potential for subject-dependent variables impacting on the regression analysis, thereby obscuring the relationship between dependent and independent variables. For this reason subjects were coded and patient dummy variables were assigned in order to negate this potential confounding factor. SPSS software, version 8.0.0 was used to perform the linear regression analysis. Evaluation of the assumptions led to no change in the planned analysis.

\section{Results}

Patient Characteristics

The age distribution of the 15 patients was $69.7 \pm 11.2$ 

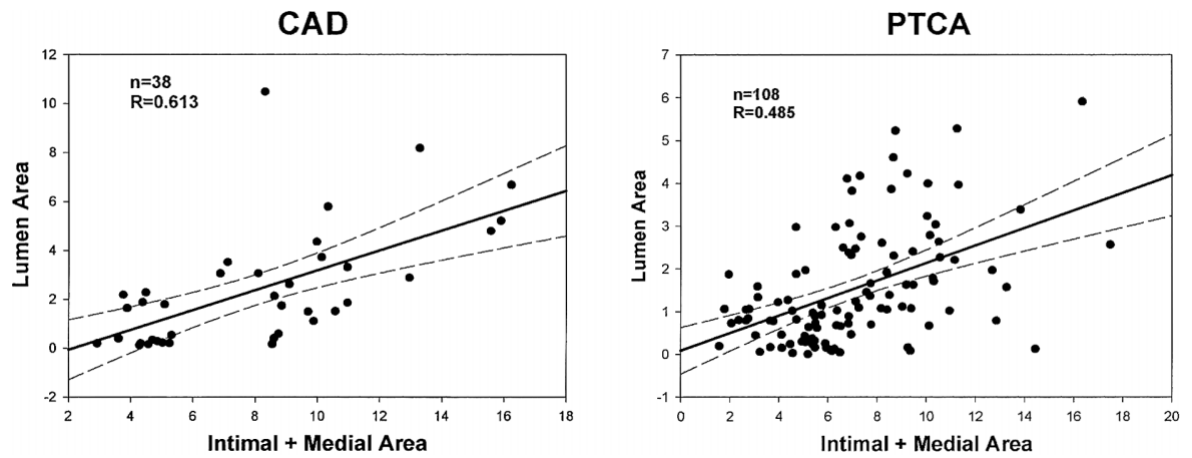

Fig 1. Relationship of lumen area (LA) vs intimal plus medial area (IA+MA) for CAD and PTCA lesions. Interrupted lines delineate $95 \%$ confidence intervals. For both groups of lesions, the LA increases as IA+MA increases; thereby indicating that compensatory enlargement occurs. However, per unit of IA+MA the magnitude of the increase in LA is less for PTCA vs CAD lesions, as the slopes of the lines are different $(\mathrm{p}=0.035)$.

Table 1 Characterization of Arterial Cross-Sections

\begin{tabular}{lcc}
\hline \hline \multirow{2}{*}{ Histological parameter } & \multicolumn{2}{c}{ Correlation with EEL index (p value) } \\
\cline { 2 - 3 } & $C A D$ & PTCA \\
\hline Adventitial inflammation & $0.55(+v e)$ & $0.32(-v e)$ \\
Adventitial microvascularity & $0.07(+v e)$ & $0.42(+v e)$ \\
Calcification & $0.42(-v e)$ & $0.61(-v e)$ \\
Eccentricity & $0.12(-v e)$ & $0.10(-v e)$ \\
EEL disruption & $0.80(+v e)$ & $0.19(+v e)$ \\
Fibrosis & $0.09(-v e)$ & $0.54(+v e)$ \\
IEL disruption & $0.22(-v e)$ & $0.86(+v e)$ \\
Intimal hyperplasia & $0.24(-v e)$ & $0.94(-v e)$ \\
Lipid & $0.28(+v e)$ & $0.14(-v e)$ \\
Medial thinning & $0.63(-v e)$ & $0.53(-v e)$ \\
Necrosis & $0.70(+v e)$ & $0.83(+v e)$ \\
Plaque inflammation & $0.29(+v e)$ & $0.78(-v e)$ \\
Plaque microvascularity & $0.69(+v e)$ & $0.04(-v e)$ \\
Thrombosis & $0.07(+v e)$ & $0.30(-v e)$ \\
\hline
\end{tabular}

EEL, external elastic lamina; $C A D$, coronary artery disease; PTCA, percutaneous transluminal coronary angioplasty; IEL, internal elastic lamina.

years (mean $\pm \mathrm{SD}$, range: $52-87$ ). There were 12 males and 3 females in the study group. The clinical indication for angioplasty was unstable angina pectoris for 3 patients, and stable angina pectoris for the remainder. The interval between coronary angioplasty and death ranged from 11 days to 4.3 years (mean: $370 \pm 438$ days). The cause of death was classified as cardiac in $12 / 15$ patients (5 patients died secondary to cardiogenic shock, 5 from refractory heart failure, 1 from a ventricular arrhythmia, and 1 from an acute myocardial infarction). Three patients died of noncardiac causes (eg, acute renal failure, gastric cancer, gastrointestinal bleeding).

\section{Coronary Arteries}

The arterial segments subjected to PTCA were greater in length than those with CAD. As a result, 108 sub-segments in 20 PTCA arterial segments were compared with 38 arterial sub-segments from $10 \mathrm{CAD}$ arterial segments. The arteries in the PTCA group were: 2 left main, 45 left anterior descending, 30 right coronary artery and 31 left circumflex. In the CAD group the arteries were: 5 left main, 14 left anterior descending, 16 right and 3 left circumflex.

\section{Quantitative Histomorphometry}

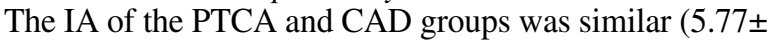

2.72 vs $6.52 \pm 3.35$, respectively; $\mathrm{p}=0.17$ ). Adding the MA to these measurements, the combined IA+MA for the PTCA and CAD groups remained similar (eg, $8.05 \pm 3.63$ vs $7.02 \pm 3.14, \mathrm{p}=0.10$ ). As well, the percentage of the IEL area that was occupied by intima or the percentage of the EEL area that was occupied by the intima plus media were similar for both the PTCA and CAD (eg, $80 \pm 13 \%$ vs $77 \pm$ $15 \%, \mathrm{p}=0.24$ and $83 \pm 11 \%$ vs $81 \pm 13 \%, \mathrm{p}=0.36$; respectively). Nonetheless, the LA of the PTCA group was smaller than that of the CAD group $\left(1.53 \pm 1.33\right.$ vs $2.40 \pm 2.40 \mathrm{~mm}^{2}$; $\mathrm{p}=0.007$ ). For both groups of specimens, compensatory enlargement occurred; that is, the LA increased as the IA+MA increased. However, the incremental increase of LA in the PTCA group was less than that of the CAD group (Fig 1). Specifically, the correlation coefficient for the graph that plots LA vs IA+MA was 0.485 in the PTCA group compared with 0.613 in the CAD group $(\mathrm{p}<0.05)$.

\section{Histological Parameters}

Fourteen histological parameters were used to characterize all arterial cross-sections (Table 1). In the PTCA group the abundance of plaque MV had a negative correlation with the EELI ( $p=0.04)$. In some arteries, plaque MV were found in all 4 quadrants of the vessel (Fig 2). However, none of the histological features of the CAD arteries corre- 

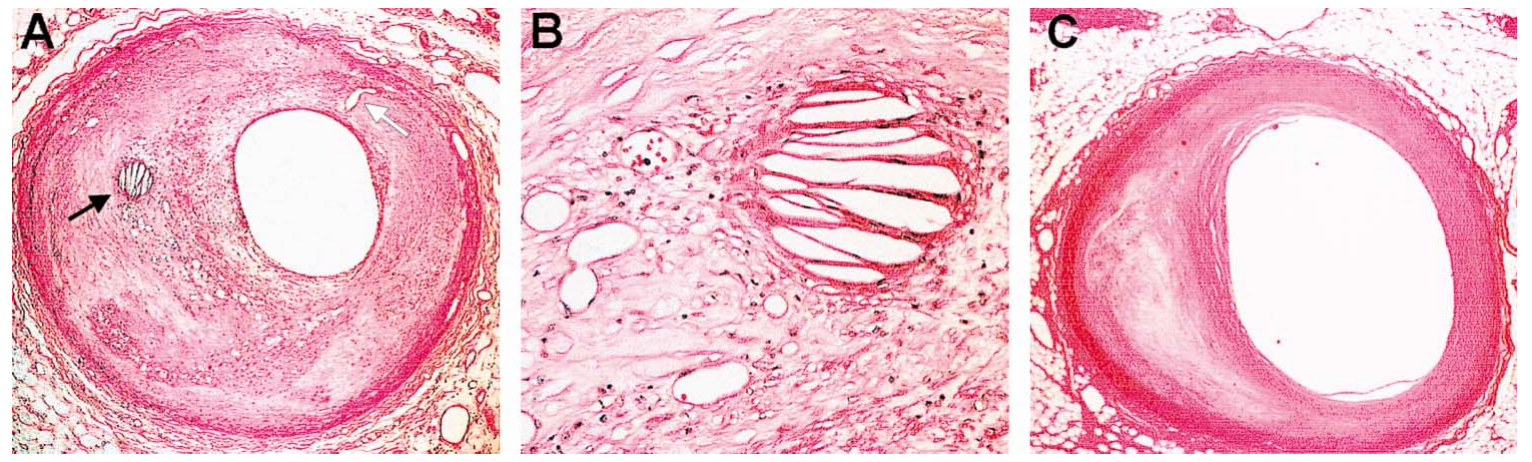

Fig 2. (A, B) Left circumflex coronary artery of a 52-year-old male who underwent angioplasty 85 days prior to death. The eccentric lesion has a low index of compensatory arterial enlargement $(\mathrm{EELI}=1.13)$. The plaque contains an abundant network of microvessels, including some larger vessels (arrow, $\times 40$ ) Panel B shows the complex web of microvessels indicated by arrow in Panel A $(\times 200)$. (C) Left anterior descending coronary artery of a 76-year-old male who underwent angioplasty 332 days ante mortem. There is compensatory enlargement (EELI $=1.64)$ and paucity of plaque microvessels. (All slides: H\&E.)

lated with the EELI. There were trends observed between a lower EELI and both the eccentricity of a lesion $(p=0.10)$ and the lipid content of the plaque $(\mathrm{p}=0.14)$. Similarly, for the $\mathrm{CAD}$ group, negative correlation trends were observed for EELI and fibrosis, as well as eccentricity ( $\mathrm{p}=0.09$ and $\mathrm{p}=0.12$; respectively). Although none of the examined parameters correlated with favorable remodeling in either the PTCA or CAD groups of lesions, there were positive correlation trends in the CAD group for the EELI and both adventitial microvascularity and thrombosis $(\mathrm{p}=0.07$ for both). As well, in the PTCA group the interval between angioplasty and death was compared with the EELI and there was a trend $(p=0.08)$ toward a negative relationship between the EELI and time post-PTCA.

\section{Discussion}

Obstruction of arterial blood flow is dependent upon the accumulation of tissue mass and the ability of the artery wall to undergo compensatory enlargement, or remodeling. In this study we focused on trying to identify specific histological features that might correlate with either favorable or unfavorable remodeling in coronary arteries that had undergone a previous PTCA. While much is known about remodeling in human $\mathrm{CAD}$ arteries and animal arteries subjected to balloon injury, we are unaware of previous histological studies that have examined the relationship between histology and remodeling in human coronary arteries subjected to PTCA. In the current study, we designated the ratio of the EEL to the sum of the plaque and medial areas (or EELI) to serve as an arbitrary, continuous variable reflective of arterial remodeling. We did not use adjacent arterial segments to make reference measurements because none of these segments were free of significant atherosclerosis.

In the PTCA lesions, we found that the abundance of plaque MV correlated with the extent of arterial constriction. In contrast, for the CAD lesions, none of the histological parameters that we studied correlated with remodeling. This observation is of particular interest because in a previous study of human coronary atherectomy specimens derived from primary and restenotic lesions we noted that intraplaque MV were more commonly found in the restenotic lesions compared with the primary specimens ${ }^{26}$ As well, the replication rate of endothelial cells that make up intraplaque MV may be remarkably elevated in some restenotic speci- mens (eg, $43.5 \%$ of all endothelial cells per tissue crosssection). Taken together, these data highlight the potential importance of the vasa vasorum in constrictive arterial remodeling. Given that nitric oxide (NO) is an endotheliumderived factor and is currently thought to be a central factor in regulating atherogenesis and vascular tone, how do we reconcile the results of the current study with the anticipated benefit that should occur with an abundance of endothelial cells in these lesions? ${ }^{27}$ Clearly, the presence of an endothelium in diseased arteries is not sufficient for a biological benefit. Several lines of evidence suggests that the endothelium of atherosclerotic arteries demonstrate a series of abnormalities, including reduced endothelialderived NO synthase (eNOS) protein expression and NO release, that result in a dysfunctional endothelium? 28,29 Therefore, delivering NO or over-expressing eNOS may be a viable means of stimulating vasorelaxation and perhaps compensatory enlargement. Consistent with these concepts are the results of the ACCORD study, which recently demonstrated that treating angioplasty patients for $12-24 \mathrm{~h}$ with NO donors results in a modest improvement in immediate and 6-month angiographic results ${ }^{30}$ Therefore, more information about the role of the endothelium and vasa vasorum in diseased arteries is needed in order to better understand both lesion development and adaptive changes in arterial wall dimensions.

\section{Study Limitations}

This study was not without limitations. Given the logistics of harvesting and perfusion fixing the arteries post mortem we were only able to assemble specimens from 15 patients and we were concerned that with this small sample size, patient specific characteristics might confound our studies. However, by incorporating patient dummy variables into the regression analysis we were able to discount the possibility that subject-dependent variables had an impact on the data analyses. As well, the relative abundance of left circumflex coronary artery segments in the PTCA group remains unexplained, but may also reflect the limitations of the small sample size. Finally, because this was an observational study we are not able to provide conclusive mechanistic insights into the biology of remodeling. To this end, we are now in the process of using animal models of arterial injury to determine exactly how the endothelium may act as a potentially important factor in remodeling post PTCA.

In conclusion, the present study determined that in 
PTCA arteries the abundance of plaque MV was associated with a lower EELI. Although PTCA is rapidly being superseded by the insertion of coronary artery stents that act as scaffolds in the artery wall and effectively minimize the importance of remodeling in lumenal renarrowing and restenosis, it is important to note that stents are not suitable for all lesions and are not free from developing restenosis. For example, in coronary arteries with diffuse disease or with a small caliber, stents may be of limited use. Therefore, targeting these types of lesions with therapeutic remodeling strategies, such as modifying endothelial function in plaque MV, may lead to the development of 'biological stenting' strategies that promote compensatory enlargement of arterial lesions?

\section{Acknowledgments}

The studies were supported by grants-in-aid to E. O'Brien from the Heart and Stroke Foundation of Canada (\#B3473, B3154) and the Medical Research Council of Canada. Dr O'Brien is a Research Scholar of the Heart and Stroke Foundation of Canada.

The authors are grateful to Valerie Duffin for assistance with the preparation of this manuscript.

\section{References}

1. Folkow B: 'Structural factor' in prmary and secondary hypertension. Hypertension 1990; 16: 89-101

2. Mann GV, Sperry A, Gray M, Jarashow D: Atherosclerosis in the Masai. Am J Epidemiol 1972; 95: 26-37

3. Glagov S, Weisenberg E, Zarins CK, Stankunavicius R, Kolettis GJ: Compensatory enlargement of human atherosclerotic coronary arteries. N Engl J Med 1987; 316: 1371-1375

4. McPherson DD, Hiratzka LF, Lamberth WC, Brandt B, Hunt M, Kieso RA, et al: Delineation of the extent of coronary atherosclerosis by high-frequency epicardial echocardiography. $N$ Engl J Med 1987; 316: $304-309$

5. Clarkson TB, Prichard RW, Morgan TM, Petrick GS, Potvin Klein K: Remodeling of coronary arteries in human and nonhuman primates. JAMA 1994; 271: 289-294

6. Isner JM: Vascular remodeling: Honey, I think I shrunk the artery. Circulation 1994; 89: 2937-2941

7. Lafont A, Guzman LA, Whitlow PL, Goormastic M, Cornhill JF, Chisolm GM: Restenosis after experimental angioplasty: Intimal, medial, and adventitial changes associated with constrictive remodeling. Circ Res 1995; 76: 996-1002

8. Kakuta T, Currier JW, Haudenschild CC, Ryan TJ, Faxon DP: Differences in compensatory vessel enlargement, not intimal formation, account for restenosis following angioplasty in the hypercholesterolemic rabbit model. Circulation 1994; 89: 2809-2815

9. Post MJ, Borst C, Kuntz RE: The relative importance of arterial remodeling compared with intimal hyperplasia in lumen renarrowing after balloon angioplasty: A study in the normal rabbit and the hypercholesterolemic Yucatan micropig. Circulation 1994; 89: 2816-2821

10. Pasterkamp G, Borst C, Post MJ, Mali WPTM, Wensing PJW, Gussenhoven EJ, et al: Atherosclerotic arterial remodeling in the superficial femoral artery: Individual variation in local compensatory enlargement response. Circulation 1996; 93: 1818-1825

11. Kimura T, Kaburagi S, Tamura T, Yokoi H, Nakagawa Y, Hamasaki $\mathrm{N}$, et al: Remodeling of human coronary arteries undergoing coronary angioplasty or atherectomy. Circulation 1999; 96: 475-483
12. Luo H, Nishioka T, Eigler NL, Forrester JS, Fishbein MC, Berglund $\mathrm{H}$, et al: Coronary artery restenosis after balloon angioplasty in humans is associated with circumferential coronary artery constriction. Arterioscler Thromb Vasc Biol 1999; 16: 1393-1398

13. Mintz GS, Popma JJ, Pichard AD, Kent KM, Satler LF, Wong SC, et al: Arterial remodeling after coronary angioplasty: A serial intravascular ultrasound study. Circulation 1996; 94: 35-43

14. Pasterkamp G, de Kleijn D, Borst C: Arterial remodeling in atherosclerosis, restenosis and after alteration of blood flow: Potential mechanisms and clinical implications. Cardiovasc Res 2000; 45: $843-852$

15. Schwartz SM, Murry CE, O'Brien ER: Vessel wall response to injury. Sci Med 1996; 32: 12-21

16. Shi Y, Pieniek M, Fard A, O'Brien J, Mannion JD, Zalewski A: Adventitial remodeling after coronary artery injury. Circulation 1996; 93: $340-348$

17. Bryant SR, Bjercke RJ, Erichsen DA, Rege A, Lindner V: Vascular remodeling in response to altered blood flow is mediated by fibroblast growth factor-2. Circ Res 1999; 84: 323-328

18. Langille BL, O'Donnell F: Reductions in arterial diameter produced by chronic diseases in blood flow are endothelium-dependent. Science 1986; 231: 405-407

19. Jamal A, Bendeck M, Langille BL: Structural changes and recovery of function after arterial injury. Arterioscler Thromb 1992; 12: $307-$ 317

20. Koester W: Endarteriitis and arteriitis. Klin Wochenschr (Berlin) 1876; 13: 454-455

21. Geiringer E: Intimal vascularization and atherosclerosis. J Pathol Bacteriol 1951; 63: 201-211

22. Heistad DH, Armstrong ML: Blood flow through vasa vasorum of coronary arteries in atherosclerotic monkeys. Arteriosclerosis 1986; 6: $326-331$

23. Labinaz M, Pels K, Hoffert C, O'Brien ER: The time course and importance of neoadventitial formation in arterial remodeling following balloon angioplasty of porcine coronary arteries. Cardiovasc Res 1999; 41: 255-266

24. Scott NA, Cipolla GD, Ross CE, Dunn B, Martin FH, Simonet L, et al: Identification of a potential role for the adventitia in vascular lesion formation after balloon overstretch injury of porcine coronary arteries. Circulation 1996; 93: $2178-2187$

25. Nobuyoshi M, Kimura T, Ohishi H, Horiuchi H, Nosaka H, Hamasaki $\mathrm{N}$, et al: Restenosis after percutaneous transluminal coronary angioplasty: Pathologic observations in 20 patients. $J$ Am Coll Cardiol 1991; 17: 433-439

26. O'Brien ER, Garvin MR, Dev R, Stewart DK, Hinohara T, Simpson $\mathrm{JB}$, et al: Angiogenesis in human coronary atherosclerotic plaques. Am J Pathol 1994; 145: 883-894

27. Schulz R, Triggle CR: Role of NO in vascular smooth muscle and cardiac muscle function. Trends Pharmacol Sci 1994; 15: 255-259

28. Wilcox JN, Subramanian RR, Sundell CL, Tracey WR, Pollock JS, Harrison DG, et al: Expression of multiple isoforms of nitric oxide synthase in normal and atherosclerotic vessels. Arterioscler Thromb Vasc Biol 1997; 17: 2479-2488

29. Oemar BS, Tschudi MR, Godoy N, Brovkovich V, Malinski T, Luscher TF: Reduced endothelial nitric oxide synthase expression and production in human atherosclerosis. Circulation 1998; 97: $2494-2498$

30. Lablanche JM, Grollier G, Lusson JR, Bassand JP, Drobinski G, Bertrand B, et al: Effect of the direct nitric oxide donors linsidomine and molsidomine on angiographic restenosis after coronary balloon angioplasty: The ACCORD study. Circulation 1998; 95: 83-89

31. Moulton KS, Heller E, Konerding MA, Flynn E, Palinski W, Folkman $\mathrm{J}$ : Angiogenesis inhibitors endostatin or TNP-470 reduce neointimal intimal neovascularization and plaque growth in apolipoprotein Edeficient mice. Circulation 1999; 99: 1726-1732 